\title{
Evaluation of Optimal Power Generation Mix Considering Nuclear Power Plants' Shut-Down Risk
}

\author{
Hiromu Matsuzawa, Ryoichi Komiyama and Yasumasa Fujii
}

\begin{abstract}
After Fukushima nuclear power plant accident, resilience engineering has emerged as a new paradigm of risk management, and the design of resilient energy system is getting more and more important. Energy model analysis based on mathematical programming contributes to discussing how to implement resilience into energy system by identifying quantitative suggestions. In this paper, as an example of such analysis, the authors try to derive possible appropriate measures to enhance electricity supply system resilience to successive nuclear power plants' shut-down risk. The model developed in this paper is a dynamic power generation planning model, which considers nuclear power plants' shut-down risk stochastically and identifies resilient capacity expansion in Japan from 2012 to 2030 under the uncertainty of the risk from a quantitative perspective. This resilient capacity expansion includes the necessity of alternative power resources and demand response compensating for supply capacity loss due to nuclear power plants' shut-down, considering economic constraints. Simulation results successfully show the need for these measures in the capacity expansion. Importantly, the suggestion is not like a future prediction but a normative image of the system through the comprehensive incorporation of forecasted future parameters and scenarios. The more detailed the parameters and the scenarios are, the better image can be obtained. Learning from past accidents and updating our scientific knowledge base will detail the parameters and the scenarios and make energy model analysis very effective. It will tell us how to make resilient energy system.
\end{abstract}

Keywords Energy model analysis - Resilient energy system • Power generation planning $\cdot$ Nuclear power plants' shut-down $\cdot$ Stochastic dynamic programming

H. Matsuzawa $(\square) \cdot$ Y. Fujii

Department of Nuclear Engineering and Management, The University of Tokyo,

Tokyo, Japan

e-mail: matsuzawa@esl.t.u-tokyo.ac.jp

R. Komiyama

Resilience Engineering Research Center, The University of Tokyo, Tokyo, Japan

(C) The Author(s) 2017

J. Ahn et al. (eds.), Resilience: A New Paradigm of Nuclear Safety,

DOI 10.1007/978-3-319-58768-4_25 


\section{Introduction}

Fukushima nuclear power plant accident has highlighted insufficient preparedness for unexpected events in many systems. Electricity supply system is one of them. By the shut-down of nuclear power plants and thermal plants in Kanto region and Tohoku region, rolling blackout was taken place to compensate for supply capacity shortage, which was the first time in Japan after the World War II. In addition, the Japanese government issued the restriction of electricity use against the large-lot electricity users in summer 2012 for the same reason. These policies and voluntary demand side management prevent massive blackout, but Japanese society had suffered heavy social and economic damages. Therefore, in the future power generation planning, preparation for power plants' successive shut-down should be considered so that social and economic damages caused by the shut-down will be the smallest. That is to say, implementation of seismic resilience into electricity supply system is necessary.

Resilience in this context refers to the adaptive capacity of a system to absorb changes and to maintain its functionality. From a quantitative perspective, resilience can be enhanced by the following three measures: "Reduces failure probability," "Reduced consequences from failures," and "Reduces time to recovery." Furthermore, enhancement measures of seismic resilience can be classified according to the following four properties [1]:

- Robustness: strength, or the ability of elements, systems, and other units of analysis to withstand a given level of stress or demand without suffering degradation or loss of function

- Redundancy: the extent to which elements, systems or other units of analysis exit that are substitutable, i.e., capable of satisfying functional requirements in the event of disruption, degradation, or loss of functionality

- Resourcefulness: the capacity to identify problems, establish priorities, and mobilize resources when conditions exit that threaten to disrupt some element, system, or other unit of analysis; resourcefulness can be further conceptualized as consisting of the ability to apply material (i.e., monetary, physical, technological, and informational) and human resources to meet established priorities and achieve goals

- Rapidity: the capacity to meet priorities and achieve goals in a timely manner in order to contain losses and avoid future disruption

Hence, seismic resilience enhancement measures in electricity supply system can be presented in accordance with these four properties. For example, robustness in electricity supply system can be enhanced by improving power plants' earthquake resistant capacity. Alternative power resources enhance redundancy. Demand side management enhances resourcefulness. Then, restoration plans enhance rapidity. Of course, it must be noted that these example are illustrative only. Many other researches which contribute the enhancement of seismic resilience also are classified into the four properties. The classification gives systematic understanding 
of the way various research activities contribute the enhancement of seismic resilience. And then, by measuring the four properties quantitatively, comprehensive coordination of those researches can be achieved.

Energy model analysis using mathematical programming is an effective tool measuring 4Rs (robustness, redundancy, resourcefulness, and rapidity) quantitatively. Then, in this paper, the authors try to present quantitative analytical framework to discuss possible appropriate measures to implement seismic resilience into electricity supply system, using simple energy model analysis. The energy model used in this paper is a dynamic power generation planning model, which incorporates the modelling of some measures in accordance with 4Rs and assesses optimal capacity expansion strategy under successive nuclear power plants' shut-down risk. It must be understood that the output of the energy model analysis should not be like the future prediction. Its major concern is not to forecast a likely future image of the energy system, but rather to derive a normative future image of the system through the comprehensive incorporation of forecasted future parameters and scenarios. The goal of this paper is to stimulate discussions about seismic resilience enhancement by the normative image obtained through energy model analysis and present its usefulness in that it can provide quantitative suggestions.

\section{Dynamic Power Generation Planning Model}

\subsection{Mathematical Formulation}

The dynamic power generation planning model mathematically expresses nuclear power plants' shut-down risk as yearly stochastic transitions of nuclear power plants availability, and identifies the optimal capacity expansion strategy under the uncertainty of the risk. This strategy is denoted by stochastic dynamic programming [Eqs. (1) and (2)] and minimizes the expected total system cost necessary from $y$ to the expiration of an analytical period. This formulation highlights that it can consider the uncertainty of both nuclear power plants' shut-down and recovery from their disruptions, so it can express the preparation for risk and adaptive methods to disruptions, which corresponds to redundancy and resourcefulness of the system.

As an analytical period, this paper assumes form 2012 to 2030, and power plants considered are thermal power (coal, LNG steam turbine (ST), LNG combined cycle (CC) and oil), nuclear power, hydro power including pumped type, and stationary sodium-sulfur battery. Exogenous variables about them are based on [2], and fuel price is set based on [3]. Concerning the installed capacity of coal-fired power plants, LNG ST power plants, oil power plants, nuclear power plants and hydro power plants, the maximum upper limit is assigned due to political, geographical or some other reasons. Regional scope is the whole region of Japan and the electricity market is assumed as a monopoly market. Annual power demand is expressed by four representative load curves of each season in 2012, and it does not change until 2030. Problem formulation is described as follows. 


\section{Endogenous variables:}

$V_{y}\left(\boldsymbol{K}_{y}, i_{y}\right) \quad$ expected total system cost $(\$)$

$C_{y}\left(\boldsymbol{K}_{y}, i, d \boldsymbol{K}_{y}\right) \quad$ yearly system cost incurred in year $y$ (\$/year)

$\boldsymbol{K}_{y} \quad$ capacity mix of power plants and power storage facilities $(\mathrm{GW})$

$K p_{y, p} \quad$ capacity of $p$-th type of power plant in year $y(\mathrm{GW})$

$d K p_{y, p} \quad$ newly constructed capacity of $p$-th type of power plant in year $y(\mathrm{GW})$

$X_{p, t, d, y} \quad$ output of $p$-th type of power plant in day $d$ at time $t$ and year $y(\mathrm{GW})$

$K s 1_{y, s} \quad \mathrm{~kW}$ capacity of $s$-th type of power storage facility in year $y(\mathrm{GW})$

$K s 2_{y, s} \quad \mathrm{kWh}$ capacity of $s$-th type of power storage facility in year $y(\mathrm{GWh})$

$d K s 1_{y, s} \quad$ newly constructed $\mathrm{kW}$ capacity of $s$-th type of power storage facility in year $y(\mathrm{GW})$

$d K s 2_{y, s} \quad$ newly constructed $\mathrm{kWh}$ capacity of $s$-th type of power storage facility in year $y(\mathrm{GWh})$

$C h a_{s, d, t, y} \quad$ input of $s$-th type of power storage facility in day $d$ at time $t$ and year $y(\mathrm{GW})$

$D i s_{s, d, t, y} \quad$ output of $s$-th type of power storage facility in day $d$ at time $t$ and year $y(\mathrm{GW})$

$S s_{s, d, t, y} \quad$ stored energy of $s$-th type of power storage facility in day $d$ at time $t$ and year $y(\mathrm{GWh})$

Save $_{d, t, y} \quad$ electricity demand saving in day $d$ at time $t$ and year $y(\mathrm{GWh})$.

where $p \in\{$ 1: Nuclear, 2: Coal, 3: LNG CC, 4: LNG ST, 5: Oil, 6: Hydro $\}, s \in\{1$ : Pumped hydro, 2: NAS battery $\}, d \in\{1,2, \ldots, 4\}, t \in\{1,2, \ldots, 24\}, y \in\{0,1, \ldots$, $18\}, i_{y}$ : state of nuclear power plants availability in year $y\left(i_{\mathrm{y}}=\{0\right.$ : nuclear power unavailable, 1: nuclear available $\}$ ).

\subsubsection{Objective Function}

Objective function is the discounted total cost considering all the possible yearly state-transitions about $i_{\mathrm{y}}$ from 2012 to 2030, which corresponds to $V_{0}\left(\boldsymbol{K}_{0}, i_{0}\right)$ in Eq. (1). Discount rate in this paper is assumed as $3 \%$. As the initial state in dynamic programming, the existing capacity in 2012 is given and nuclear power plants are assume to be available [Eq. (4)].

$V_{y}\left(\boldsymbol{K}_{y}, i_{y}\right)=\min _{d \boldsymbol{K}_{y}}\left\{C_{y}\left(\boldsymbol{K}_{y}, i_{y}, d \boldsymbol{K}_{y}\right)+\exp (-\gamma) \sum_{i_{y+1}=0}^{1} P\left(i_{y} \rightarrow i_{y+1}\right) V_{y+1}\left(\boldsymbol{K}_{y+1}, i_{y+1}\right)\right\}$

$$
V_{19}\left(\boldsymbol{K}_{19}, i_{19}\right)=0
$$




$$
\begin{gathered}
\boldsymbol{K}_{y+1}=\boldsymbol{K}_{y}+d \boldsymbol{K}_{y}+\boldsymbol{d e c} \boldsymbol{c}_{y} \\
\left(\boldsymbol{K}_{0}, i_{0}\right)=(\boldsymbol{k i n i}, 1) \\
\boldsymbol{K}_{y}=\left(K p_{y, 1}, K p_{y, 2}, \ldots, K p_{y, 6}, K s 1_{y, 1}, K s 1_{y, 2}, K s 2_{y, 1}, K s 2_{y, 2}\right)
\end{gathered}
$$

where, $\boldsymbol{d e c}_{y}$ : decommission capacity, $\boldsymbol{k i n i}$ : existing capacity in $2012, \gamma$ : discount rate, $P(\cdot)$ : state transition probability

$$
\begin{gathered}
C_{y}\left(\boldsymbol{K}_{y}, i_{y}, d \boldsymbol{K}_{y}\right)=\sum_{p=1}^{6}\left(g_{p} \times p f_{p} \times d K p_{y, p}+\frac{365}{4} \sum_{d=1}^{4} \sum_{t=1}^{24} p v_{p, y} \times X_{p, t, d, y}\right) \\
+\sum_{s=1}^{2} C S_{s, y}+\frac{365}{4} \sum_{d=1}^{4} \sum_{t=1}^{24} \operatorname{Csave}_{d, t, y} \\
C S_{s, y}=g s 1_{s} \times p f s 1_{s} \times d K s 1_{s}+g s 2_{s} \times p f s 2_{s} \times d K s 2_{s}+p f s 3_{s, y} \times \frac{\text { TCh }_{s, y}}{\text { cycle }_{s}} \\
T C h a_{s, y}=\frac{365}{4} \sum_{d=1}^{4} \sum_{t=1}^{24} C h a_{s, d, t, y}
\end{gathered}
$$

where $g_{p}$ : annual fixed charge rate of $p$-th type of power plant (capital recovery factor), $p f_{p}$ : unit fixed cost of $p$-th type of power plant $(\$ / \mathrm{kW}), p v_{p, y}$ : unit variable cost of $p$-th type of power plant $(\$ / \mathrm{kWh}), C S_{s, y}$ : annual cost of $s$-th type of power storage facility, $g s 1_{s}$ : annual fixed charge rate for power component of $s$-th type of power storage facility, $p f s 1_{s}$ : unit fixed cost for power component of $s$-th type of power storage facility (cost for $\mathrm{kW}$ capacity, $\$ / \mathrm{kW}$ ), $g s 2_{s}$ : annual fixed charge rate for energy component of $s$-th type of power storage facility, $p f s 2_{s}$ : unit fixed cost for energy component of $s$-th type of power storage facility (cost for $\mathrm{kWh}$ capacity, $\$ / \mathrm{kWh}$ ), pfs $3_{s, y}$ : unit fixed cost for consumable material of $s$-th type of power storage facility $(\$ / \mathrm{kWh})$, cycle $_{s, y}$ : maximum recharge times of $s$-th type of power storage facility, $T C h a_{s, y}$ : annual total charged electricity of $s$-th type of power storage facility(kWh/year).

The fourth item in right hand side of Eq. (6) is demand saving cost, which is mathematically modelled as the penalty cost incurred by economic loss based on [4]. This paper assumes typical demand curves (Fig. 1) where reference price is $P_{0}$ and reference demand power load in day $d$ at time $t$ and year $y$ is $\operatorname{load}_{d, t, y}$. According to this curve, the promotion of energy saving from reference point cause the escalation of electricity price, and eventually, the integral of the demand curve from reference demand $\operatorname{load}_{d, t, y}$ to curtailed demand $\operatorname{load}_{d, t, y}$ minus Save d,t,y $_{\text {cor- }}$ responds to the penalty cost, which is formulated in Eq. (9) or (10). Variable Save $_{d, t, y}$ is endogenously determined through cost minimization considering the cost competitiveness towards the capacity expansion cost. This modelling methods of supply shortage depends on reference price $P_{0}$ and price elasticity. In this paper, 
Fig. 1 Electricity demand curve and energy saving

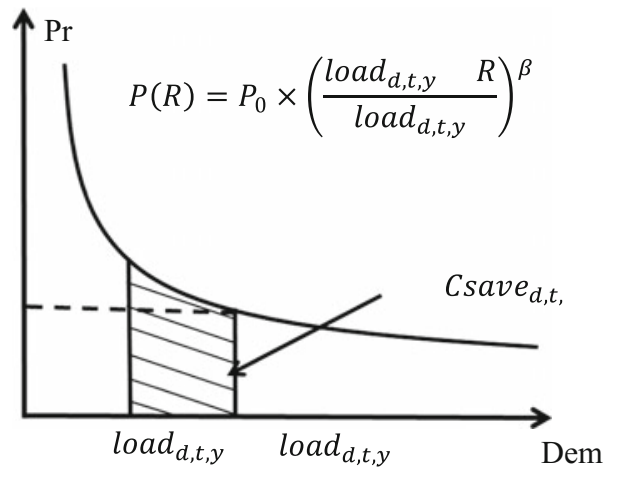

$P_{0}$ is assumed as the shadow price of electricity demand in dynamic power generation planning model that does not consider the nuclear power plants' shut-down risk, and price elasticity is assumed as $0.1 \%$. From systemic view, the modelling of demand saving corresponds to that of resourcefulness.

$$
\begin{aligned}
\text { Csave }_{d, t, y} & =\int_{0}^{\text {Save }_{d, t, y}} P(R) d R=\int_{0}^{\text {Save }_{d, t, y}} P_{0} \times\left(\frac{\text { load }_{d, t, y}-R}{\operatorname{load}_{d, t, y}}\right)^{-\frac{1}{\beta}} d R \\
& =\frac{\beta}{1-\beta} \times \operatorname{load}_{d, t, y} \times P_{0} \times\left\{\left(\frac{\operatorname{load}_{d, t, y}-\text { Save }_{d, t, y}}{\operatorname{load}_{d, t, y}}\right)^{\frac{\beta-1}{\beta}}-1\right\} \quad(\beta \neq 1) \\
\text { Csave }_{d, t, y} & =\int_{0}^{\operatorname{Save}_{d, t, y}} P(R) d R=\int_{0}^{\operatorname{Save}_{d, t, y}} P_{0} \times\left(\frac{\text { load }_{d, t, y}-R}{\operatorname{load}_{d, t, y}}\right)^{-\frac{1}{\beta}} d R \\
& =\operatorname{load}_{d, t, y} \times P_{0} \times \log \left(\frac{\text { load }_{d, t, y}-\operatorname{Save}_{d, t, y}}{\operatorname{load}_{d, t, y}}(\beta=1)\right.
\end{aligned}
$$

where $\beta$ : price elasticity, $P_{0}$ : reference price, $\operatorname{load}_{d, t, y}$. power demand in day $d$ at time $t$ and year $y(\mathrm{GWh})$.

\subsubsection{Constraints}

The dynamic power generation planning model minimizes total expected cost under various technical constraints employing linear programming technique. The constraints considered in this paper are based on [2], and formulated as follows: 
(a) Available capacity constraint of the plants

$$
X_{p, t, d, y} \leq a v p_{d, p}\left(i_{y}\right) \times K p_{y, p}
$$

where $\operatorname{avp}_{d, p}\left(i_{y}\right)$ : availability factor of $i$-th type of power plant.

(b) Power demand and supply balances

$$
\sum_{p=1}^{6} X_{p, t, d, y}+\sum_{s=1}^{2}\left(\operatorname{Dis}_{s, d, t, y}-\text { Cha }_{s, d, t, y}\right)=\operatorname{load}_{d, t, y}-\text { Save }_{d, t, y}
$$

(c) Constraint on installable capacity

$$
\begin{aligned}
K p_{y, p} & \leq K \operatorname{Pmax}_{y, p} \\
K s 1_{y, s} & \leq K S 1 \max _{y, s} \\
K s 2_{y, s} & \leq K S 2 \max _{y, s}
\end{aligned}
$$

where $K P \max _{p}$ : maximum installable capacity of $p$-th type of power plant in year $y(\mathrm{GW}), K S 1 \max _{y, s}$ : maximum installable $\mathrm{kW}$ capacity of $s$-th type of power storage facility in year $y(\mathrm{GW}), K S 2 \max _{y, s}$ : maximum installable kWh capacity of $s$-th type of power storage facility in year $y(\mathrm{GWh})$.

(d) Constraint on load following capability of the plants

$$
\begin{aligned}
& X_{p, t+1, d, y} \leq X_{p, t, d, y}+\text { increase }_{p} \times \operatorname{avp}_{d, p}\left(i_{y}\right) \times K p_{y, p} \\
& X_{p, t+1, d, y} \geq X_{p, t, d, y}-\text { decrease }_{p} \times \operatorname{avp}_{d, p}\left(i_{y}\right) \times K p_{y, p}
\end{aligned}
$$

where increase $_{p}$ : maximum output increase rate per unit time of $p$-th type of power plant, decrease $e_{p}$ : maximum output decrease rate per unit time of $p$-th type of power plant.

(e) Charge and discharge balances of energy storage technology

$$
S s_{s, d, t+1, y}=\left(1-s d_{s}\right) \times S s_{s, d, t, y}+\left(\sqrt{e f f_{s}} \times C h a_{s, d, t, y}-\frac{D i s_{s, d, t, y}}{\sqrt{e f f_{s}}}\right)
$$

where $s d_{s}$ : self-discharge rate of $s$-th type of power storage facility, eff $f_{s}$ cycle efficiency of electricity storage of $s$-th type of power storage facility. 
(f) Available capacity constraint of the battery technology

$$
\begin{gathered}
C h a_{s, d, t, y}+D i s_{s, d, t, y} \leq u s 1_{s, d} \times K s 1_{y, s} \\
S s_{s, d, t, y} \leq u s 2_{s} \times K s 2_{y, s} \\
S s_{s, d, t, y} \leq m_{s} \times u s 1_{s} \times K s 1_{y, s}
\end{gathered}
$$

where $u s 1_{s}: \mathrm{kW}$ availability factor of $s$-th type of power storage facility, $u s 2_{s}: \mathrm{kWh}$ availability factor of $s$-th type of power storage facility, $m_{s}$ : energy storage capacity per generation capacity of $s$-th type of power storage facility $(\mathrm{kWh} / \mathrm{kW})$.

\subsection{Nuclear Power Plants' Shut-Down Model}

In this paper, nuclear power plants' shut-down risk is mathematically expressed as yearly stochastic transitions of nuclear power plants availability based on [5]. The methodologies are as follows.

Suppose normal state means nuclear power plants are available and accident state means unavailable, disruption rate $\lambda$, the rate of disruption occurrence per time step, is formulated by the reliability of nuclear power plants availability $R$ [Eq. (22)]. If disruption rate $\lambda$ is independent from time steps, disruption density function $f(y)$, which means the rise rate of unreliability $1-R$, is denoted by Eqs. (23) and (24). Mean time between disruptions (MTBD), the expected mean time nuclear power plants can continue their operation, is obtained [Eq. (25)], and mean time to recovery (MTTR), the expected mean time nuclear power plants restart their operation after disruption, is formulated in a similar way through the definition of recovery rate $\mu$. State transition probability between normal state and accident state, which corresponds to unreliability $1-R$, is determined by MTBD and MTTR. This paper assumes MTBD and MTTR as 30 years and 2 years respectively. In this model, MTBD and MTTR represents the robustness and the rapidity of the system.

$$
\begin{gathered}
\lambda=-\frac{1}{R} \frac{d R}{d y} \\
R=\exp (-\lambda y) \\
f(y)=\frac{d(1-R)}{d y}=\lambda \exp (-\lambda y) \\
M T B D=\int_{0}^{\infty} y f(y) d y=\frac{1}{\lambda}
\end{gathered}
$$




$$
\begin{gathered}
\text { MTTR }=\frac{1}{\mu} \\
P(0 \rightarrow 1)=1-\exp (-1 / \text { MTTR }) \\
P(1 \rightarrow 0)=1-\exp (-1 / M T B D)
\end{gathered}
$$

where $\lambda$ : disruption rate, $R$ : the reliability of nuclear power plants' availability, $f(y)$ : disruption density function, MTBD: mean time between disruptions (year), MTTR: mean time to recovery (year).

\subsection{Calculation Algorithm}

To solve strictly this model formulated using stochastic dynamic programming needs a lot of computations because of the high dimensionality of $\boldsymbol{K}_{y}$, and calculation is difficult due to computational constraints. This problem is called "the curse of dimensionality". Therefore, as an approximate solution method to stochastic dynamic programming, cutting planes method [6] is adopted in this paper. This method uses the convexity of $V_{y}\left(\boldsymbol{K}_{y}, i_{y}\right)$, which characteristic is due to the linear programming technique employed in Eqs. (1)-(28), and $V_{y}\left(\boldsymbol{K}_{y}, i_{y}\right)$ is approximated as a set of hyper planes defined at sample points $\boldsymbol{K}_{y}^{*}$ on the function described in Fig. 2 and Eq. (29). The approximation allow us to solve this model at each time step while it is necessary to solve at one time considering all the time steps during analytical period if you want to solve this model strictly. It achieves a lot of computational saving, and can successfully address the curse of dimensionality. The detailed algorithm of cutting planes method is shown in Fig. 3.

$$
V_{y}\left(\boldsymbol{K}_{y}, i_{y}\right) \geq V_{y}\left(\boldsymbol{K}_{y}^{*}, i_{y}\right)+\frac{\partial V_{y}\left(\boldsymbol{K}_{y}^{*}, i_{y}\right)}{\partial \boldsymbol{K}_{y}}\left(\boldsymbol{K}_{y}-\boldsymbol{K}_{y}^{*}\right)
$$

where $\boldsymbol{K}_{y}^{*}$ : a sample point defined on $V_{y}\left(\boldsymbol{K}_{y}, i_{y}\right)$.

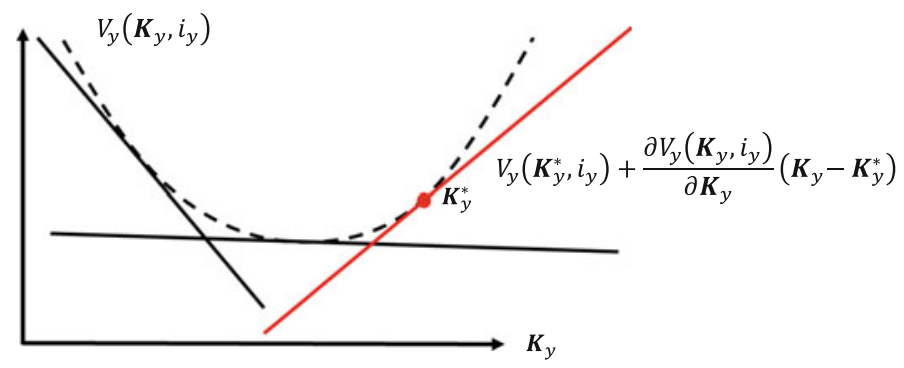

Fig. 2 Approximation by cutting planes method 


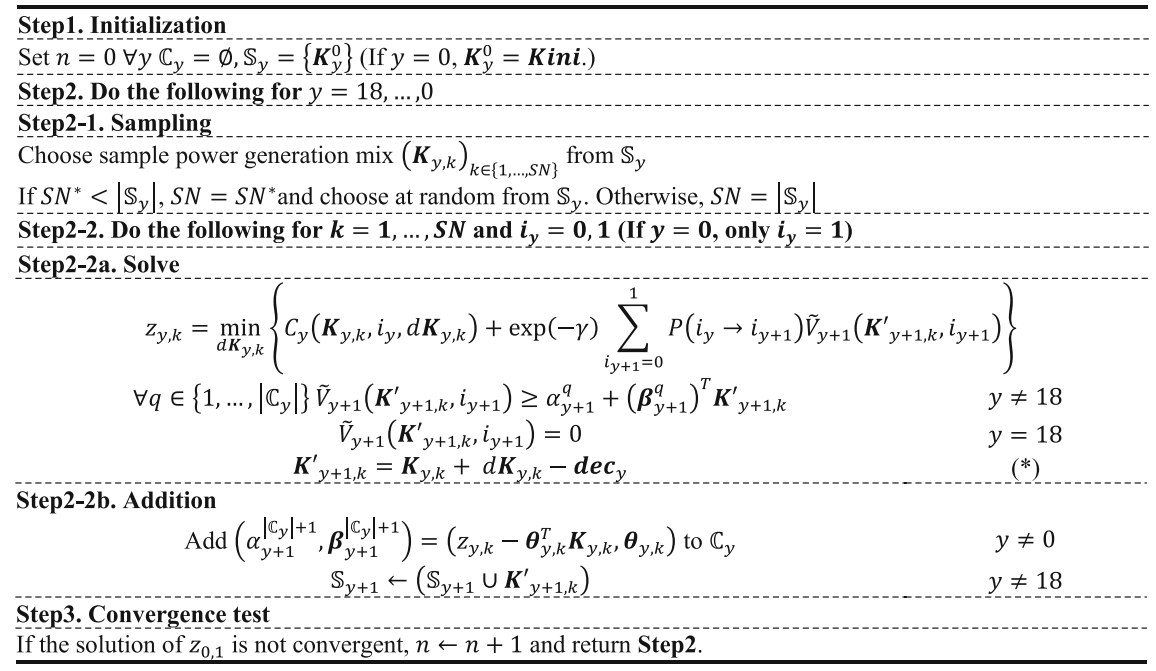

Fig. 3 Cutting planes method algorithm

where $n$ : iteration number, $\boldsymbol{K}_{y}^{0}$ : initial sample point (arbitrarily set except in case $y=0), \mathbb{C}_{y}$ : constraint space in year $y, \mathbb{S}_{y}:$ sample point space in year $y, \boldsymbol{K}_{y, k}:$ chosen sample points, $S N$ : sampling number, $S N^{*}$ : maximum sampling number, $\tilde{V}_{y+1}$ : approximated function of $V_{y+1}, \boldsymbol{\theta}_{y, k}$ : shadow price of equation (*) in step 2-2a.

\section{Results and Discussion}

The algorithm shown in Fig. 3 allow us to simulate any scenarios by solving the problem forwardly from $y=0$ to $y=18$. This paper presents two representative scenarios. The first scenario (Scenario 1) assumes the shut-down does not happen during the time period. In the second scenario (Scenario 2), the shut-down happens in 2026 and it recovers 2028. In addition to the two scenarios, reference case, where the shut-down risk is zero, is calculated.

Figure 4 shows estimated capacity mix in reference case and Scenario 1. In scenario 1, the LNG CC capacity is expanded at larger scale. It can be said that the uncertainty of nuclear power plants' shut-down encourages to have redundancy in electricity supply system.

Figure 5 shows the comparison of supply capacity in Scenario 1 and Scenario 2, and Fig. 6 shows the estimated daily power generation dispatch in summer 2026, 

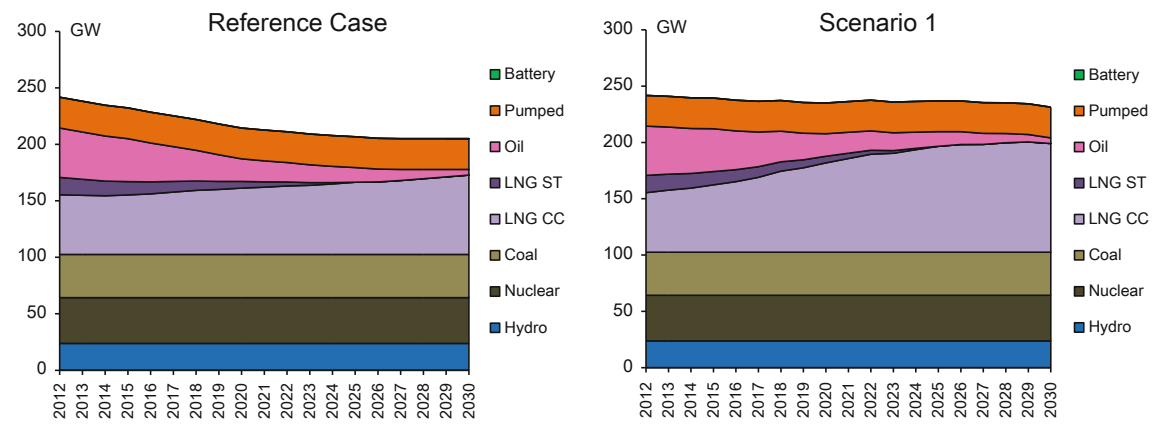

Fig. 4 Estimated capacity mix from 2012 to 2030

Fig. 5 Power supply capacity

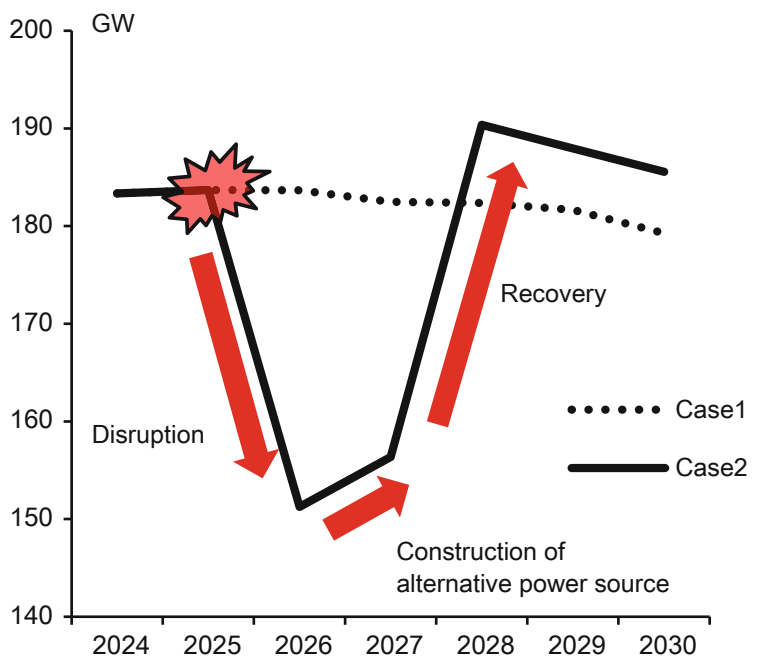

when nuclear power plants' shut-down happens. Immediately after disruption, demand saving happens at peak time (11:00-18:00), and then, the construction of alternative power source can been seen in 2027 to compensate for the supply capacity loss. These policies highlight the resourcefulness of the system to addresses the nuclear power plants' shut-down. It should be noted that the construction of alternative power source minimizes the resilient triangle expressed in Fig. 5 under economic constraints. 


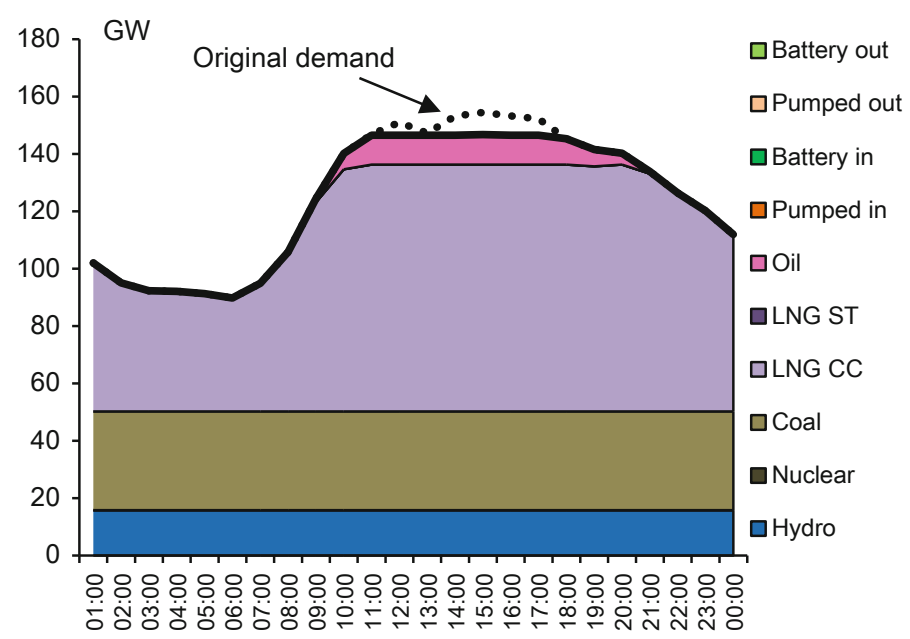

Fig. 6 Daily power generation dispatch in 2026 (Scenario 2)

\section{Conclusions and Future Works}

This paper presents a quantitative analytical framework to assess seismic resilient enhancement measures in electricity supply system, referring to the four essential properties; robustness, redundancy, resourcefulness and rapidity. The dynamic power generation planning model considering nuclear power plants' shut-down risk successfully derives possible appropriate measures to enhance resilience of the system from a quantitative perspective, and gives systemic understanding of the system's seismic resilience. Simulation results show that nuclear power plants' shut-down risk creates a need for redundancy in power generation planning and that the decrease of electricity supply capacity caused by nuclear power plants' shut-down can be compensated for by demand saving and construction of alternative power source. In other words, it can be compensated for by the system's resourcefulness.

As mentioned in introduction, the simulation results highlight a normative image of the system through the comprehensive incorporation of forecasted future parameters and scenarios. However, the model developed in this paper is a work in progress and not simple enough to do so. To get better understanding of seismic resilience, our future works consists in refining the model and consider such parameters and scenarios. With respect to the refinement of parameters and scenarios, it depends on technical and social researches. For example, MTTR can be shortened through enhancement of quake-resistance and tsunami protection of nuclear power plants. It can also shortened through good design of social systems 
where effective decision making is done even under extreme situations, and effective decontamination actions. The value of MTBD can be assessed more precisely by geologically-based study. Price elasticity, which determines the resourcefulness of the system, will be lowered aggressive introduction of emergency power source although cost-effectiveness of their installation should be considered, of course. Assessment on these policies through energy model analysis will give the potential contributions and benefits of them. Concerning scenarios planning and model refinement, the design of systems has a key role. Social activities in today's world are supported by highly complex and interdependent system, and so, risks surrounding us are very systemic. Fukushima nuclear power plant accident is such a kind of risk. Therefore, targeted system should be comprehensive enough to consider their inter-relations although the system considered in this paper is limited within electricity supply system. In addition, more kinds of risks should be considered because our challenges to be dealt with now in this world are not only nuclear usage.

Finally, to address above requirements, the most important seems to learn effectively from accidents and update our social scientific knowledge base. The implement of what we have learned into energy model makes it more sophisticated and the model will tell us how to design resilient systems.

Acknowledgements This work was supported by JST Strategic Basic Research Programs RISTEX, Resilience Analysis for Social Safety Policy.

\section{References}

1. M. Bruneau et al., A framework to quantitatively assess and enhance the seismic resilience of communities. Earthquake Spectra 19, 733-752 (2003)

2. R. Komiyama, Y. Fujii, Long-term scenario analysis of nuclear energy and variable renewables in Japan's power generation mix considering flexible power resources. Energy Policy 83, 169-184 (2015)

3. International Energy Agency (IEA), World Energy Outlook 2013 (OECD, Paris, France, 2013)

4. H. Matsuzawa, R. Komiyama, Y. Fujii, Analysis of energy system resilience to disaster in Kanto Region using stochastic programming. Proc. Conf. Energy Econ. Environ. 34, 223-226 (2015)

5. Y. Uchiyama, Y. Hatano, K. Okajima, Social Risk in Energy System (CORONA Publishing Co., Ltd., 2012), pp. 70-74

6. Z.L. Chen, W.B. Powell, Convergent cutting plane and partial-sampling algorithm for multistage stochastic linear programs with recourse. J. Optim. Theory Appl. 102, 497-524 (1999) 
Open Access This chapter is licensed under the terms of the Creative Commons Attribution 4.0 International License (http://creativecommons.org/licenses/by/4.0/), which permits use, sharing, adaptation, distribution and reproduction in any medium or format, as long as you give appropriate credit to the original author(s) and the source, provide a link to the Creative Commons license and indicate if changes were made.

The images or other third party material in this chapter are included in the chapter's Creative Commons license, unless indicated otherwise in a credit line to the material. If material is not included in the chapter's Creative Commons license and your intended use is not permitted by statutory regulation or exceeds the permitted use, you will need to obtain permission directly from the copyright holder. 